\title{
Higher tumor mass and lower adipose mass are associated with colon-26 adenocarcinoma-induced cachexia in male, female and ovariectomized mice
}

\author{
TAYLOR BANH $^{1}$, DEENA SNOKE ${ }^{1}$, RACHEL M. COLE ${ }^{1}$, AUSTIN ANGELOTTI ${ }^{1}$, \\ PATRICK M. SCHNELL ${ }^{2}$ and MARTHA A. BELURY ${ }^{3}$ \\ ${ }^{1}$ Interdisciplinary PhD Program in Nutrition, The Graduate School; \\ ${ }^{2}$ Division of Biostatistics, College of Public Health; ${ }^{3}$ Department of Human Sciences, \\ College of Education and Human Ecology, The Ohio State University, Columbus, OH 43201, USA
}

Received May 22, 2018; Accepted September 19, 2018

DOI: 10.3892/or.2019.7079

\begin{abstract}
Cachexia is responsible for nearly $20 \%$ of all cancer-related deaths, yet effective therapies to prevent or treat the disease are lacking. Clinical studies have shown that male patients lose weight at a faster rate than females. Additionally, an 'obesity paradox' may exist where excess adiposity may confer survival to patients with cancer cachexia. To further explore these phenomena, the aim of this study was to evaluate the role of changes of adipose tissue mass, sex status, and tumor mass on outcomes of male, female and ovariectomized (OVX) mice with C-26 adenocarcinoma-induced cachexia. We used EchoMRI to assess body composition and grip strength to measure muscle function. Body weights and food intake were measured daily. Mice were euthanized 19 days postinoculation. Post-necropsy, muscle fiber cross-sectional areas were quantified and real-time PCR was performed for genes relating to proteolysis. Survival curve, correlation and multiple linear regression analyses were performed to identify predictors of cachexia. Female and OVX tumor mice developed cachexia similarly to males, as evidenced by loss of skeletal and adipose masses, decreased grip strength, and increased proteolytic gene expression. Notably, female and OVX tumor mice had earlier onset of cachexia ( $\geq 5 \%$ weight loss) than
\end{abstract}

Correspondence to: Dr Martha A. Belury, Department of Human Sciences, College of Education and Human Ecology, The Ohio State University, Campbell Hall, 1787 Neil Ave, Columbus, OH 43201, USA E-mail: belury.1@osu.edu

Abbreviations: C-26, colon-26; OVX, ovariectomized; RPMI, Roswell Park Memorial Institute; PBS, phosphate-buffered saline; CSA, cross-sectional area; OCT, optimal cutting temperature; H\&E, hematoxylin and eosin; SEM, standard error of the mean; ANOVA, analysis of variance; UPS, ubiquitin proteasome system

Key words: sex, ovariectomy, adipose, C-26 adenocarcinoma, cancer cachexia male tumor mice. Larger tumor mass and lower adipose mass were the strongest predicting factors for increased severity of cachexia, regardless of sex or ovariectomy status. These results indicated that the impact of sex status may be subtle in comparison to the predictive effect of tumor and adipose mass in mice with C-26-induced cachexia.

\section{Introduction}

Cancer cachexia is a multifactorial disease characterized by ongoing loss of skeletal muscle mass (with or without adipose mass) that leads to functional impairment (1) and contributes to $20 \%$ of all cancer-related deaths (2). Since much of the research in cancer cachexia has historically focused on muscle loss, the role of adipose in this disease is less understood. The loss of adipose mass often precedes the loss of lean (i.e., muscle) mass in cancer cachexia and can predict for mortality in cancer patients (3-7). Similar to other wasting diseases like end-stage chronic kidney disease and chronic heart failure (8), an 'obesity paradox' may exist in patients with cancer cachexia, where obese individuals develop cachexia at a slower rate than lean patients (9). Possible explanations for this obesity paradox may relate to adipose tissue's potential protective effects, including: i) acting as a large energy reservoir in a state of negative energy balance and ii) secreting important adipokines for regulating whole-body metabolism and inflammation.

Several clinical studies have reported that male cancer patients lose weight at a faster rate than females (10-13), although explanations for this finding are lacking. In the general population, females tend to have $\sim 10 \%$ more adiposity than men at the same BMI (14), which may fit the obesity paradox idea in patients with cancer cachexia. The role of menopause, a condition associated with an increase in adipose mass (15), is also unclear in cancer cachexia.

Understanding the interactions between adiposity and sex as contributors to the pathogenesis of cancer cachexia could aid in developing more effective therapies for patients. However, findings from clinical studies are often complex and difficult to interpret due to the heterogeneity of cancer types and potential 
pre-disposing factors in patients. To circumvent these issues, studies aiming to understand the development and progression of cancer cachexia often utilize rodent models. The colon-26 (C-26) adenocarcinoma model is a well-characterized model of cancer cachexia due to its ability to mimic many of the human pathophysiology and underlying molecular mechanisms driving the disease (16). Like humans, C-26 tumor-bearing mice exhibit reduced body mass (e.g., muscle and adipose loss), systemic inflammation and activation of ubiquitin ligases in skeletal muscle which leads to protein degradation $(17,18)$. The functional impairments of muscle (e.g., muscle strength and force, respiratory failure, heart failure) observed in patients with cancer cachexia are also reproduced in C-26 tumor-bearing mice $(19,20)$. Anorexia, another important factor that contributes to body wasting in people (21), has been reported in C-26 tumor-bearing mice (22). The phenotypes of the C-26 tumor mouse model are highly reproducible and provide researchers a standardized model that can be used to elucidate mechanisms that contribute to cachexia and develop pre-clinical therapies that may potentially slow or reverse the disease.

The aim of this study was to evaluate the changes of adipose tissue mass, sex status and tumor mass on outcomes of mice with C-26 adenocarcinoma-induced cachexia. Our study revealed that female and ovariectomized (OVX) mice developed cachexia sooner than male mice and that independent of sex, this finding was related to higher tumor mass and lower adipose mass predicting for the onset and severity of weight loss.

\section{Materials and methods}

Colon-26 adenocarcinoma cell culture. C-26 adenocarcinoma cells were cultured with Roswell Park Memorial Institute media (RPMI 1640) + L-glutamine (Sigma-Aldrich; Merck KGaA, Darmstadt, Germany) supplemented with 5\% fetal bovine serum and $1 \%$ penicillin-streptomycin at $37^{\circ} \mathrm{C}$ and $5 \% \mathrm{CO}_{2}$.

Experimental animals and study design. CD2F1 mice ( $\mathrm{n}=20$ five-week old males, $\mathrm{n}=20$ nine-week old intact females, $\mathrm{n}=20$ nine-week old OVX females) of similar weight $(\sim 20 \mathrm{~g})$ were purchased from Charles River (Wilmington, MA, USA). Mice were housed in a vivarium equipped with room temperature of $22 \pm 0.5^{\circ} \mathrm{C}$, a 12 -h light/dark cycle, and free access to food and water. Upon arrival, mice were housed in groups of 5 mice per cage and allowed 1 week to acclimate to environmental conditions. On the day of tumor cell inoculation (day 0), half of the mice in each group (male, female, OVX) were subcutaneously injected into the right flank with $1 \times 10^{6} \mathrm{C}-26$ cells suspended in $100 \mu \mathrm{l}$ phosphate buffered saline (PBS). An equal volume of PBS was injected into the right flank of mice serving as controls for each of the 3 groups. The weight of all mice in the study ranged from $18.9-21.5 \mathrm{~g}$ at day 0 . Bodyweight and food intake of mice were measured daily. Mice were euthanized in a fed state $(\sim 1300 \mathrm{~h})$, by heart puncture under anesthesia with cervical dislocation at 19 days post-inoculation. Tissues and blood were immediately collected and prepared for analyses as described below. Experiments involving mice were approved by The Ohio State University Institutional Animal Care and Use Committee.
EchoMRI. To assess time-course changes in body composition in live mice, EchoMRI ${ }^{\mathrm{TM}}$ (Houston, TX, USA) was used at 3 time-points: $24 \mathrm{~h}$ prior to tumor cell inoculation, day 8 post-inoculation and days 17-19 post-inoculation (day of necropsy).

Grip strength. Mice were acclimated to forelimb and hindlimb grip strength testing for 1 week and grip strength was measured beginning on day 7 post-inoculation, followed by days 14 and 19 post-inoculation (day of necropsy) with the Columbus Instruments Grip Strength Meter (Columbus, $\mathrm{OH}$, USA). Forelimb and hindlimb grip strength of each mouse were tested 3 times consecutively with 1 min rest between replicates; the replicate average was used for analysis.

Muscle fiber cross-sectional area. To determine muscle fiber size via cross-sectional area (CSA), freshly isolated gastrocnemius samples were mounted with Optimal Cutting Temperature (OCT) compound, and snap-frozen with liquid nitrogen-cooled isopentane. Mounted samples were sectioned using a cryostat (Leica; Wetzlar, Hesse, Germany). Three serial sections (10 $\mu \mathrm{m})$ spanning the length of each gastrocnemius muscle were prepared and stained with hematoxylin and eosin (H\&E). Images were acquired using an Olympus IX71 microscope and cellSens Standard software (Center Valley, PA, USA). Muscle fiber CSA was quantified by fiber diameter (ImageJ; National Institutes of Health, Bethesda, MD, USA). An evaluator manually outlined and quantified individual fibers for a separate blinded evaluator, who then grouped results accordingly for data analysis. Results from each of the 3 sections per muscle were averaged prior to statistical analysis.

ELISA. Plasma IL-6 (Invitrogen; Thermo Fisher Scientific, Inc., Waltham, MA, USA) and adiponectin (EMD Millipore, Billerica, MA, USA) were measured by ELISA according to the manufacturers' protocol.

Real-time quantitative PCR. Muscle RNA was extracted with TRIzol (Invitrogen; Thermo Fisher Scientific, Inc.) according to the manufacturer's protocol. RNA concentration was determined (NanoDrop 1000; Thermo Fisher Scientific, Inc.) and RNA quality was confirmed by $1 \%$ agarose gel. RNA was reverse transcribed to cDNA (High Capacity cDNA Archive Kit, Applied Biosystems; Thermo Fisher Scientific, Inc.). The cDNA was amplified by real-time quantitative PCR with TaqMan Gene Expression Assays using pre-designed and validated primers under universal cycling conditions defined by Applied Biosystems (Thermo Fisher Scientific, Inc.) The primers used were MuRF-1 (product ID code: Mm01185221_m1), Atrogin-1 (product ID code: Mm00499523_m1) and Bax (product ID code:Mm00432051_m1)obtainedfrom ThermoFisherScientific, Inc. The thermocycling conditions were: $95^{\circ} \mathrm{C}$ for $10 \mathrm{~min}$, followed by 40 cycles at $95^{\circ} \mathrm{C}$ for $15 \mathrm{sec}$ and $60^{\circ} \mathrm{C}$ for $1 \mathrm{~min}$. Target gene expression was normalized to the endogenous control GAPDH (product no. 4352339E; Thermo Fisher Scientific, Inc.) amplified in the same reaction and expressed as $2^{-\mathrm{DDCq}}$ relative to the control group (23).

Statistical analysis. Data are presented as the mean \pm standard error of the mean (SEM). Plasma IL-6 and adiponectin data 
Table I. Characteristics of male, female, and OVX tumor mice.

\begin{tabular}{|c|c|c|c|c|c|c|}
\hline & \multicolumn{2}{|c|}{ Male } & \multicolumn{2}{|c|}{ Female } & \multicolumn{2}{|c|}{ OVX } \\
\hline & Control & Tumor & Control & Tumor & Control & Tumor \\
\hline Tumor-free necropsy body weight (g) & $22.5 \pm 0.5$ & $18.0 \pm 0.5^{\mathrm{a}}$ & $20.4 \pm 0.2$ & $15.3 \pm 0.4^{\mathrm{a}}$ & $22.8 \pm 0.6$ & $17.3 \pm 0.7^{\mathrm{a}}$ \\
\hline Cumulative food intake (g/mouse) & $33.2 \pm 0.2$ & $27.2 \pm 1.5$ & $27.6 \pm 1.0$ & $23.3 \pm 1.2$ & $30.5 \pm 1.4$ & $25.5 \pm 2.0$ \\
\hline Quadriceps muscle (mg) & $168 \pm 2$ & $133 \pm 4^{\mathrm{a}}$ & $155 \pm 2$ & $117 \pm 5^{\mathrm{a}}$ & $167 \pm 4$ & $130 \pm 5^{\mathrm{a}}$ \\
\hline Gastrocnemius muscle (mg) & $137 \pm 3$ & $113 \pm 4^{\mathrm{a}}$ & $123 \pm 3$ & $101 \pm 4^{\mathrm{a}}$ & $129 \pm 3$ & $115 \pm 5$ \\
\hline Soleus muscle (mg) & $6.5 \pm 0.3$ & $4.8 \pm 0.3^{\mathrm{a}}$ & $6.1 \pm 0.3$ & $5.3 \pm 0.4$ & $6.3 \pm 0.4$ & $4.5 \pm 0.5^{\mathrm{a}}$ \\
\hline Muscle fiber CSA $\left(\mu \mathrm{m}^{2}\right)$ & $1850 \pm 102$ & $1542 \pm 119$ & $2187 \pm 72$ & $1283 \pm 118^{a}$ & $2104 \pm 215$ & $1492 \pm 60$ \\
\hline Inguinal adipose (mg) & $563 \pm 61$ & $209 \pm 68^{a}$ & $437 \pm 38$ & $67 \pm 19^{\mathrm{a}}$ & $710 \pm 52$ & $191 \pm 44^{\mathrm{a}}$ \\
\hline Epididymal adipose (mg) & $451 \pm 30$ & $125 \pm 42^{\mathrm{a}}$ & $292 \pm 20$ & $60 \pm 10^{\mathrm{a}}$ & $418 \pm 35$ & $110 \pm 22^{\mathrm{a}}$ \\
\hline Heart (mg) & $125 \pm 5$ & $130 \pm 7$ & $107 \pm 3$ & $105 \pm 4$ & $113 \pm 3$ & $111 \pm 7$ \\
\hline Liver (mg) & $765 \pm 16$ & $899 \pm 28^{a}$ & $730 \pm 24$ & $809 \pm 50$ & $825 \pm 10$ & $871 \pm 26$ \\
\hline Spleen (mg) & $59 \pm 2$ & $206 \pm 11^{\mathrm{a}}$ & $80 \pm 4$ & $160 \pm 15^{\mathrm{a}}$ & $75 \pm 2$ & $190 \pm 13^{\mathrm{a}}$ \\
\hline LOG plasma IL-6 (pg/ml) & $1.2 \pm 0.2$ & $2.5 \pm 0.2^{\mathrm{a}}$ & $1.5 \pm 0.1$ & $2.5 \pm 0.1^{\mathrm{a}}$ & $1.1 \pm 0.2$ & $2.4 \pm 0.1^{\mathrm{a}}$ \\
\hline LOG plasma adiponectin $(\mu \mathrm{g} / \mathrm{ml})$ & $1.0 \pm 0.0$ & $0.5 \pm 0.1^{\mathrm{a}}$ & $1.1 \pm 0.0$ & $0.5 \pm 0.1^{\mathrm{a}}$ & $1.0 \pm 0.0$ & $0.7 \pm 0.0^{\mathrm{a}}$ \\
\hline
\end{tabular}

andicates significantly different from respective control by Student's t-test with Bonferroni correction $(\alpha=0.05 / 3, \mathrm{P}<0.016)$. Data are expressed as the mean \pm SEM; $n=8-10$ mice/group for all measurements, except, cumulative food intake $n=2$ cages/group and muscle fiber CSA $\mathrm{n}=3-5$ mice/group. OVX, ovariectomized; CSA, cross-sectional area.

were log-transformed to reduce skewedness of distribution. Differences between male, female, and OVX tumor groups were analyzed by one-way analysis of variance (ANOVA) followed by post-hoc Tukey's test. Differences between control and tumor groups were analyzed by Student's t-test with Bonferroni correction for multiple hypothesis testing $(\alpha=0.05 / 3)$. A one-sample t-test with Bonferroni correction was used to test for differences between beginning and final measurements within a single group. Pearson's correlation and multiple linear regression analyses were used to determine interactions of variables and outcomes in the experimental model. All statistical tests were performed using the software GraphPad Prism 7 (GraphPad Software, Inc., La Jolla, CA, USA) with the exception of multiple linear regression analysis, which was performed with R (Vienna, Austria). All tests were performed at the 5\% significance level, adjusting for multiplicity where noted.

\section{Results}

Characterization of $C-26$ cachexia between male, female and OVX mice. By 19 days post-inoculation, all tumor-bearing mice in the male, female and OVX groups developed cachexia as defined by $\geq 5 \%$ weight loss (Fig. $1 \mathrm{~A}$ and $\mathrm{B}$ ); tumor-free necropsy body weight was significantly smaller in all tumor-bearing groups compared to their respective control groups at the end of the study (Table I, $\mathrm{P}<0.016$ ). Three female tumor mice and two OVX tumor mice were euthanized prior to day 19 post-inoculation (i.e., days 17 and 18), due to early body weight loss $>20 \%$ of peak body weight and signs of moribundity (e.g., diminished movement, anorexia). When tumor mass was normalized to tumor-free bodyweight at necropsy, female tumor mice had significantly higher tumor mass per gram of bodyweight compared to male and OVX tumor mice
(Fig. 1C, $\mathrm{P}=0.012$ and $\mathrm{P}<0.001$ vs. male and $\mathrm{OVX}$ tumor mice, respectively). Anorexia (e.g., decreased food intake) was evident in all tumor-bearing groups (Fig. 1D), and cumulative food intake was lower compared to their respective control groups (Table I). To assess changes in body composition over time, EchoMRI analysis was performed on mice before tumor inoculation, at midpoint (day 8 post-inoculation) and the day of necropsy (days 17-19 post-inoculation). There were no changes in lean body mass (Fig. 1E), while adipose mass was reduced at the end of the study in all 3 tumor groups (Fig. 1F, $\mathrm{P}<0.016)$. Female tumor mice lost significantly more adipose mass (expressed as the percentage change of initial) than OVX tumor mice at the end of the study (Fig. $1 F, P=0.036$ ).

Effect of C-26 cachexia on skeletal muscle tissue. Despite no significant changes in lean body mass as measured by EchoMRI (Fig. 1E), all tumor groups experienced decreases in skeletal muscle masses compared to their respective control groups (Table I, $\mathrm{P}<0.016$ ). There were no significant differences in skeletal muscle masses among the 3 tumor groups (Fig. 2A). Similarly, there were no significant differences in average muscle fiber cross-sectional area (Fig. 2B and C), although female tumor mice had a significantly higher $\%$ of small fibers (range $0-499 \mu \mathrm{m}^{2}$ ) than male tumor mice (Fig. 2D, $\mathrm{P}=0.03$ ). As a measurement of muscle function, grip strength was measured at days 7,14 and 19 post-inoculation. Data were normalized to day 7 post-inoculation (before onset of cachexia), when peak forelimb grip strength was observed in all 3 tumor groups. OVX tumor mice had significantly decreased forelimb grip strength at the end of the study (Fig. 2E, $\mathrm{P}<0.016$ ), while female tumor mice exhibited a similar trend $(\mathrm{P}=0.026)$. The change in forelimb grip strength was not significantly different between groups at the end of the study $(\mathrm{P}=0.104$ and $\mathrm{P}=0.075$ for female and $\mathrm{OVX}$ tumor mice vs. male tumor 
A

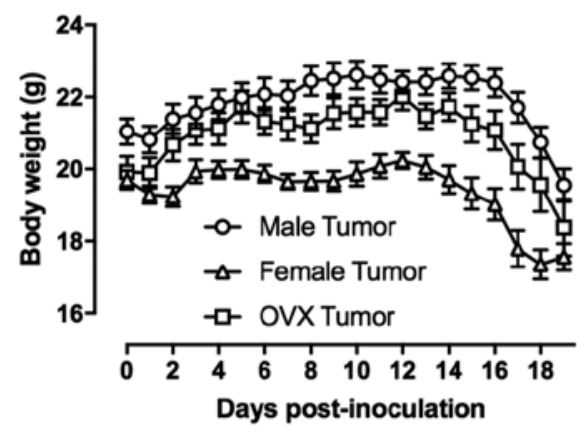

C

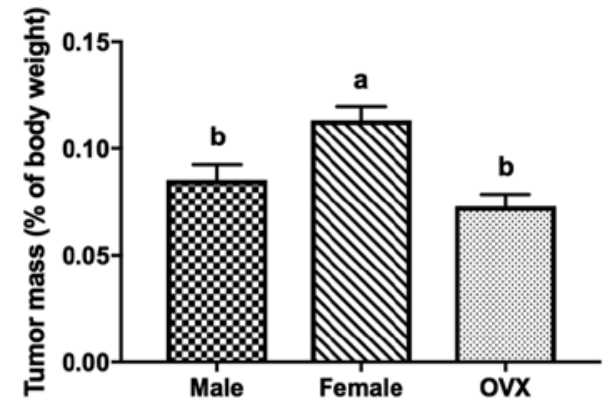

E

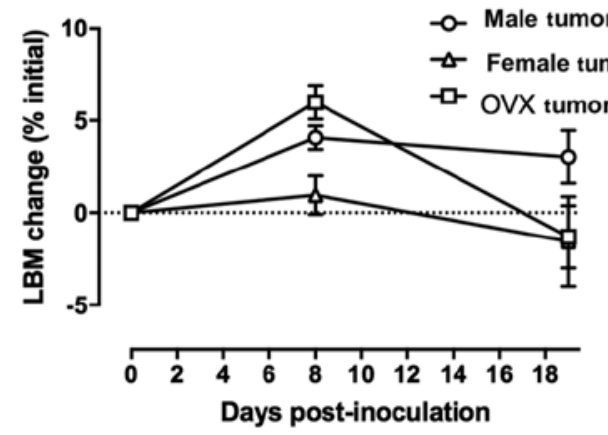

B
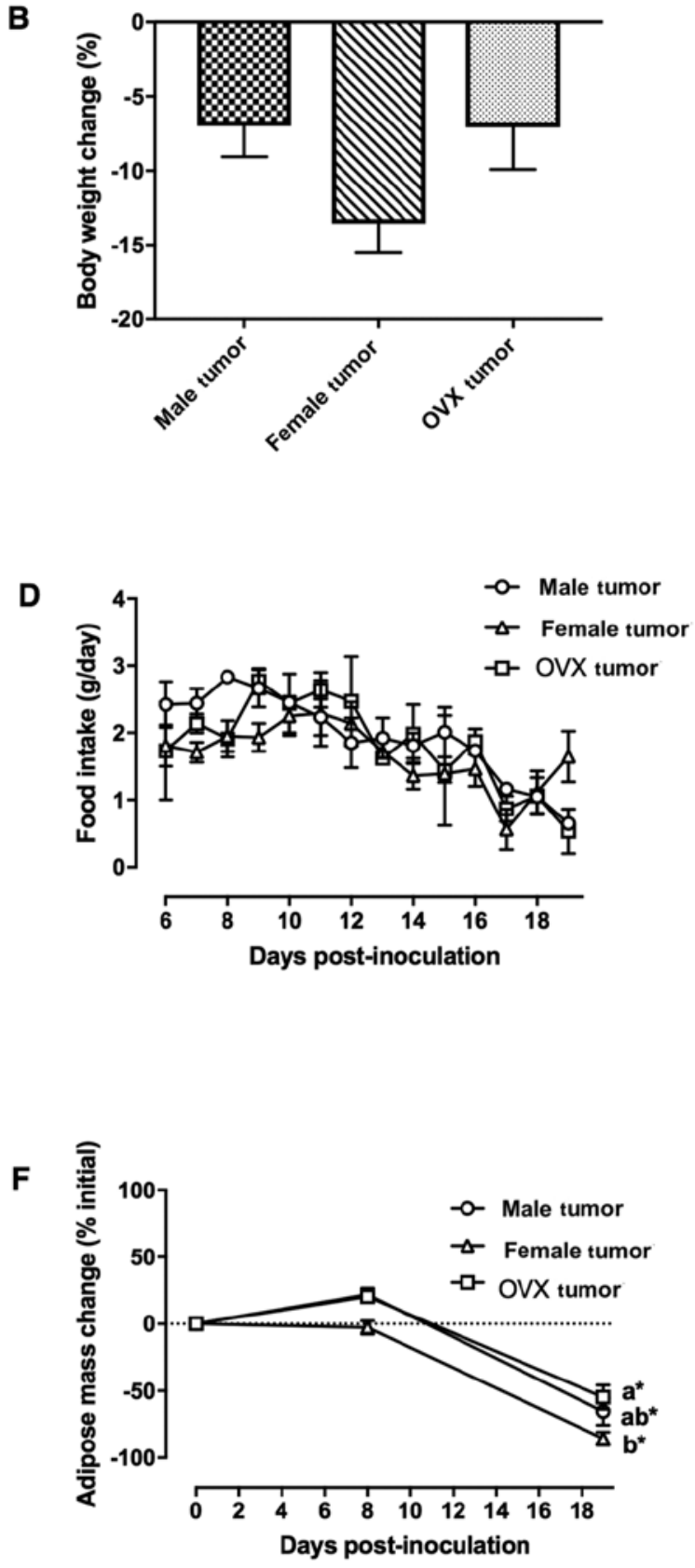

Figure 1. Characteristics of C-26 tumor-bearing male, female and OVX mice. (A) Daily body weights of tumor-bearing mice over the 19-day experimental period. (B) C-26 tumor cell inoculation induced body weight loss in all mice at necropsy. (C) Tumor mass expressed as a percentage of tumor-free body mass in mice. (D) Food intake of tumor mice. Food intake was calculated by dividing total food consumed each day by the number of mice in each cage $(n=2$ cages/group). (E and F) Lean body mass and adipose mass changes of tumor mice over the experimental period. Data are expressed as the mean \pm SEM ( $\mathrm{n}=9-10 /$ group, unless otherwise indicated). Significant differences $(\mathrm{P}<0.05)$ are represented by different letters $(\mathrm{a}, \mathrm{b})$ using one-way ANOVA with post-hoc Tukey's test. Significant differences $(\mathrm{P}<0.016)$ between day 0 and day 19 within a single group are represented by $*($ Fig. $1 \mathrm{~F}$, all 3 groups). $\mathrm{C}-26$, colon-26; OVX, ovariectomized.

mice, respectively). There were no significant differences in hindlimb grip strength between the 3 tumor groups (Fig. 2F). The C-26 model of cancer cachexia is known for its aggressive ability to induce muscle wasting. The primary mechanism responsible for C-26-induced muscle wasting is through the ubiquitin proteasome system (UPS) (24). Consistent with other studies using the C-26 model of cancer cachexia (25-27), male, female and OVX tumor groups exhibited significant increases in mRNA levels of E3 ubiquitin ligases Atrogin-1 and MuRF-1 in quadriceps muscle in comparison to their respective controls (data not shown); however, these genes were not significantly different between the 3 tumor groups (Fig. 2G). Another mechanism involved in cancer-induced muscle wasting is through $\mathrm{Bcl}$-2-like protein 4 (Bax)-regulated apoptosis (18). While Bax mRNA levels were significantly higher in the 3 tumor groups in comparison to their respective controls (data not shown), they were not significantly different between the 3 tumor groups (Fig. 2G).

Effect of C-26 cachexia on non-skeletal muscle tissues and plasma biomarkers. Male, female and OVX tumor groups exhibited significant decreases in both inguinal (subcutaneous) 
A
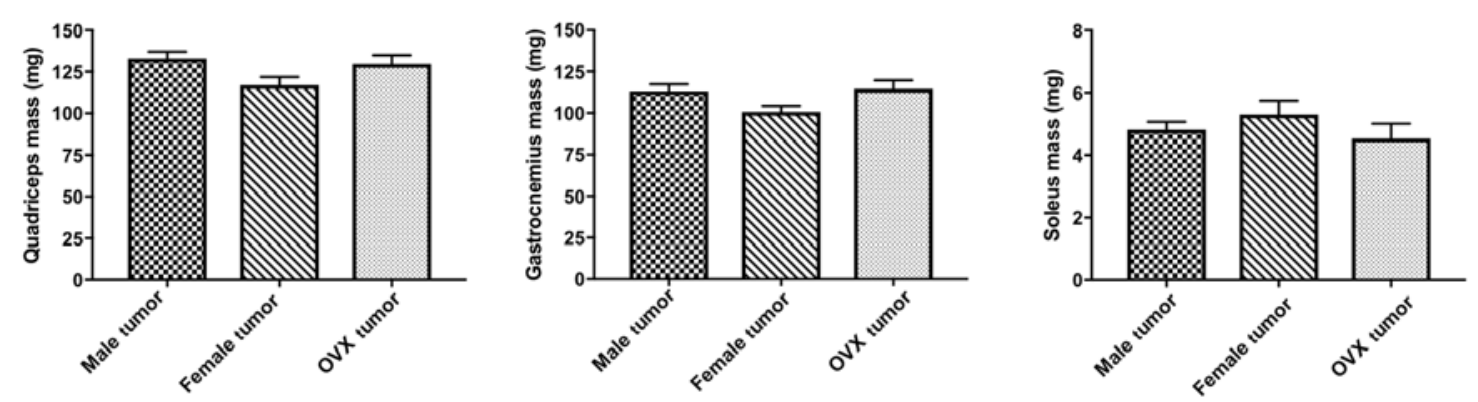

B
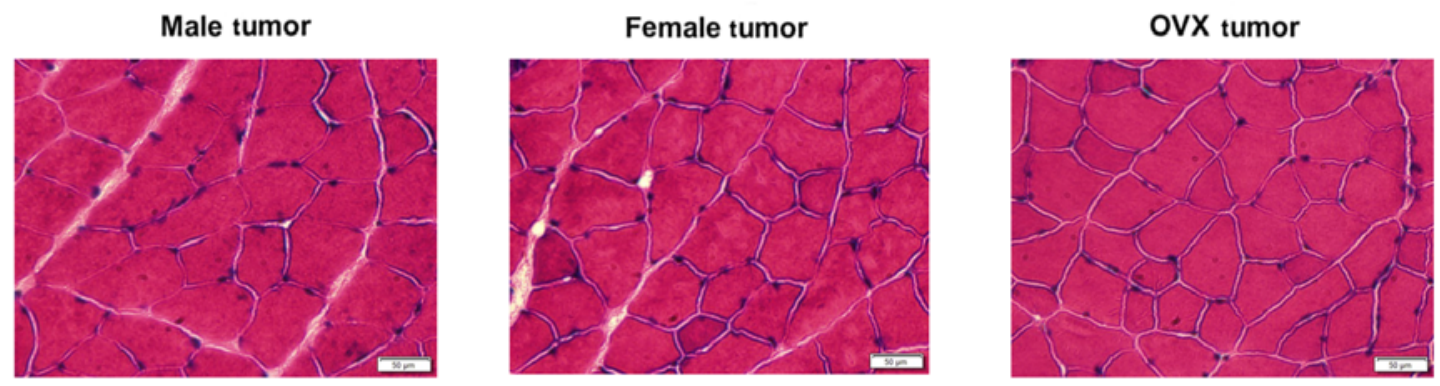

C

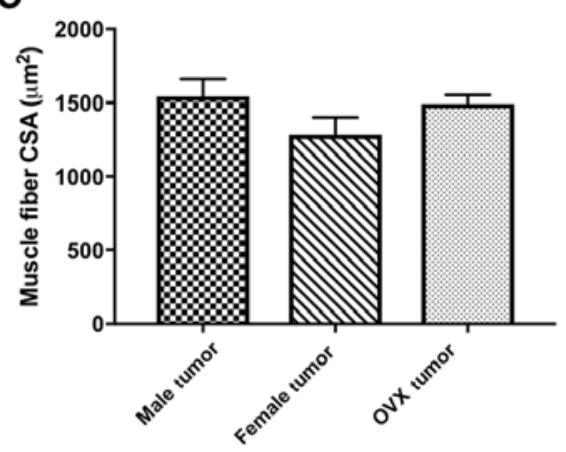

D

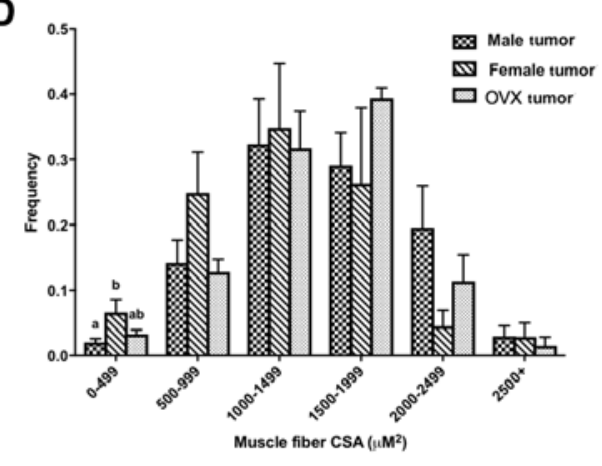

E

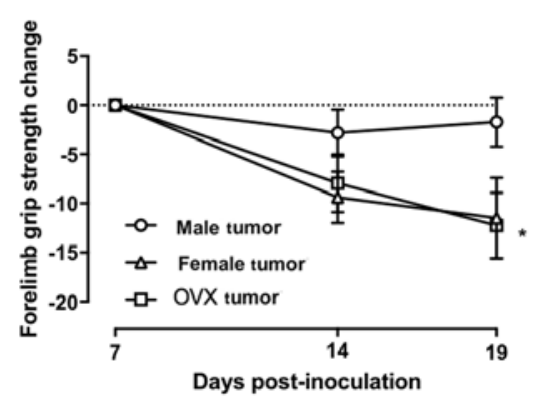

F

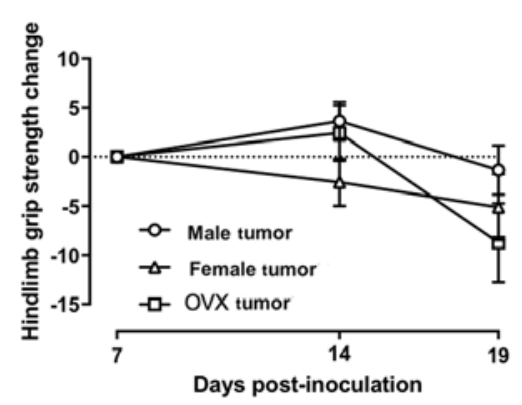

G

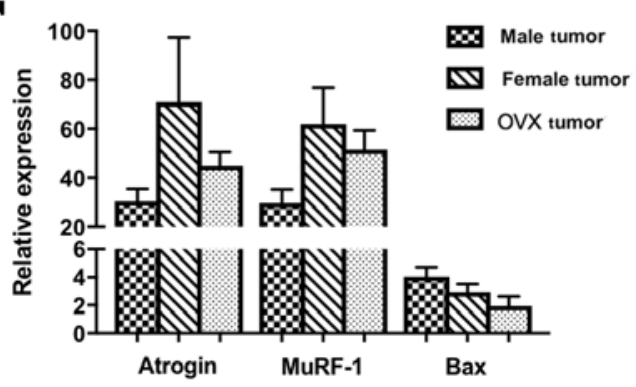

Figure 2.C-26 cachexia induces loss of muscle mass and function in male, female and OVX mice. (A) Muscle weights of tumor mice at necropsy. (B) Hematoxylin and eosin staining of representative gastrocnemius sections of tumor mice (magnification, x20; scale bar, $50 \mu \mathrm{m}$ ). (C) Quantification of gastrocnemius CSA ( $n=3-5 /$ group). (D) Frequency distribution of gastrocnemius CSA (n=3-5/group). (E and F) Change in forelimb and hindlimb grip strength of tumor mice from peak grip strength (day 7 post-inoculation). (G) Relative gene expression of markers related to muscle wasting; relative fold change was normalized to male control mice, which was set at 1 (data not shown). Data are expressed as mean \pm SEM ( $n=8-10$ /group unless otherwise indicated). Significant differences $(\mathrm{P}<0.05)$ are represented by different letters $(\mathrm{a}, \mathrm{b})$ using one-way ANOVA with post-hoc Tukey's test. Significant differences $(\mathrm{P}<0.016)$ between Day 7 and Day 19 within a single group are represented by * as observed in E, OVX tumor. C-26, colon-26; OVX, ovariectomized. 
A

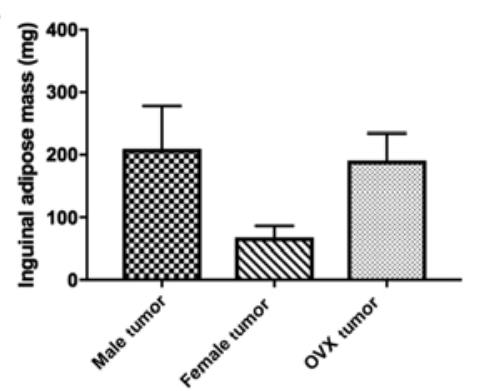

B

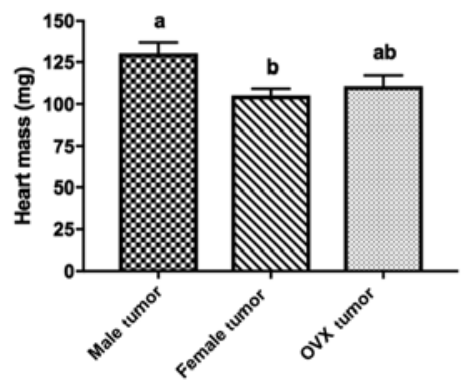

C

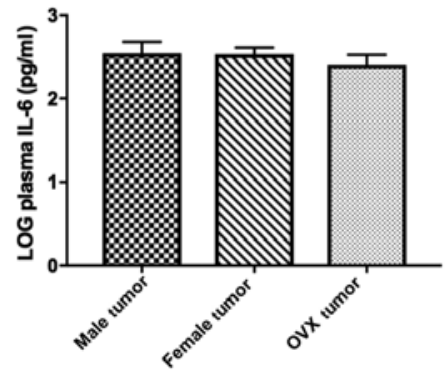

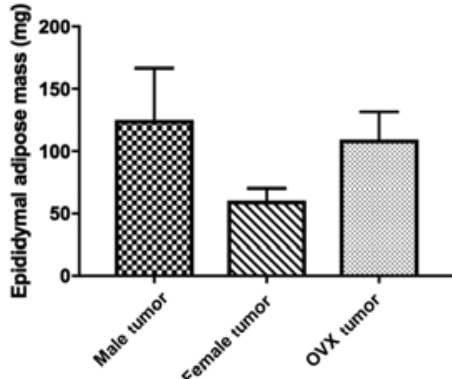
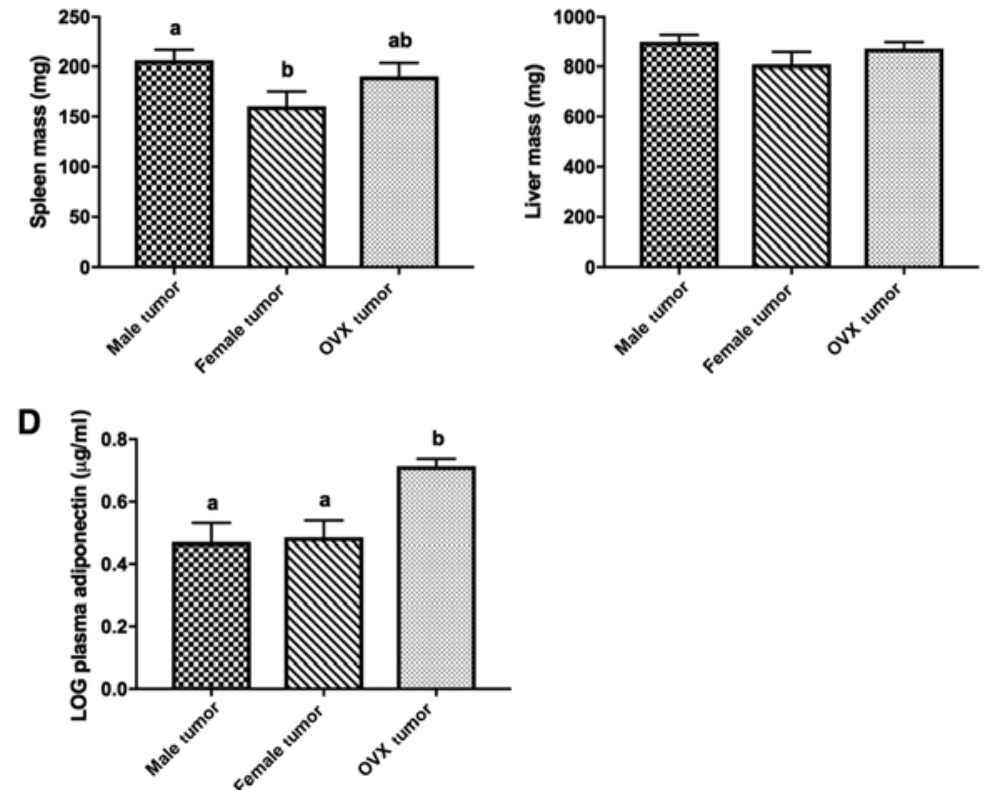

Figure 3. C-26 cachexia induces adipose loss, increases plasma IL-6 and decreases plasma adiponectin in male, female and OVX mice. (A) Adipose depot weights of tumor mice at necropsy. (B) Heart, liver and spleen weights of tumor mice at necropsy. (C) Plasma IL-6 levels of tumor mice at necropsy. (D) Plasma adiponectin levels of tumor mice at necropsy. Data are expressed as the mean $\pm \mathrm{SEM}(\mathrm{n}=8-10 /$ group). Significant differences $(\mathrm{P}<0.05)$ are represented by different letters $(\mathrm{a}, \mathrm{b})$ using one-way ANOVA with post-hoc Tukey's test. C-26, colon-26; OVX, ovariectomized.

and epididymal (visceral) adipose depots compared to their respective controls (Table $\mathrm{I}, \mathrm{P}<0.016$ ), but there were no significant differences between the 3 tumor groups (Fig. 3A). Heart and spleen masses were significantly lower in female tumor mice compared to male tumor mice (Fig. 3B, $\mathrm{P}=0.014$ and $\mathrm{P}=0.048$, respectively), but this was not observed in OVX tumor mice. Liver mass was similar between the 3 tumor groups. IL-6 is the primary cytokine responsible for inducing cachexia in the C-26 models (17). Male, female, and OVX tumor groups had significantly higher levels of plasma IL-6 compared to their respective controls (Table I, $\mathrm{P}<0.016$ ), but these values were not significantly different between the 3 tumor groups (Fig. 3C). Adiponectin, a hormone with whole-body anti-inflammatory and insulin-sensitizing roles (28) was significantly decreased in all tumor groups compared to their respective controls (Table $\mathrm{I}, \mathrm{P}<0.016$ ); plasma adiponectin was significantly higher in OVX tumor mice compared to male tumor and female tumor mice (Fig. 3D, $\mathrm{P}=0.004$ and 0.005 vs. male and female tumor mice, respectively).

Relationship of tumor and adipose mass to the progression of $\mathrm{C}-26$ cachexia. Although the 3 tumor groups developed cachexia ( $\geq 5 \% \mathrm{BW}$ loss) by the end of the study, the rate of developing cachexia differed among groups (Fig. 4A,
$\mathrm{P}=0.04)$. Female and OVX tumor mice (50\% incidence at days 14.5 and 15.5 post-inoculation, respectively) appeared to develop cachexia earlier than male tumor mice (day 17.5 postinoculation). To discern the possible reason(s) for differences in susceptibility to developing cachexia, we performed correlational analyses of potential factors that could contribute to body weight loss. Tumor mass (expressed as tumor mass normalized to tumor-free body mass) was correlated with percentage of peak body weight loss (Fig. 4B, $\mathrm{P}<0.001$ ), regardless of sex or ovariectomy. Initial mouse body weight and lean body mass (as measured by EchoMRI) did not predict for overall body weight loss or adipose loss (data not shown). EchoMRI adipose mass at midpoint (day 8 post-inoculation, before mice developed cachexia) predicted for overall percentage of adipose loss at the end of the study (Fig. 4C, $\mathrm{P}=0.025$ ). Since adipose loss in tumor mice may be impacted by tumor mass, multiple linear regression analysis was performed using percentage of tumor mass and EchoMRI adipose mass (g) as co-variates for overall percentage of adipose loss (Fig. 4D). There was a significant effect of the percentage of tumor mass on the percentage of adipose loss $(\mathrm{P}=0.022)$, when controlling for EchoMRI adipose mass (g) (Table II). A 1 unit increase in the percentage of tumor mass was associated with a 4.0 percentage point loss of adipose mass. There was not a significant effect of 
A

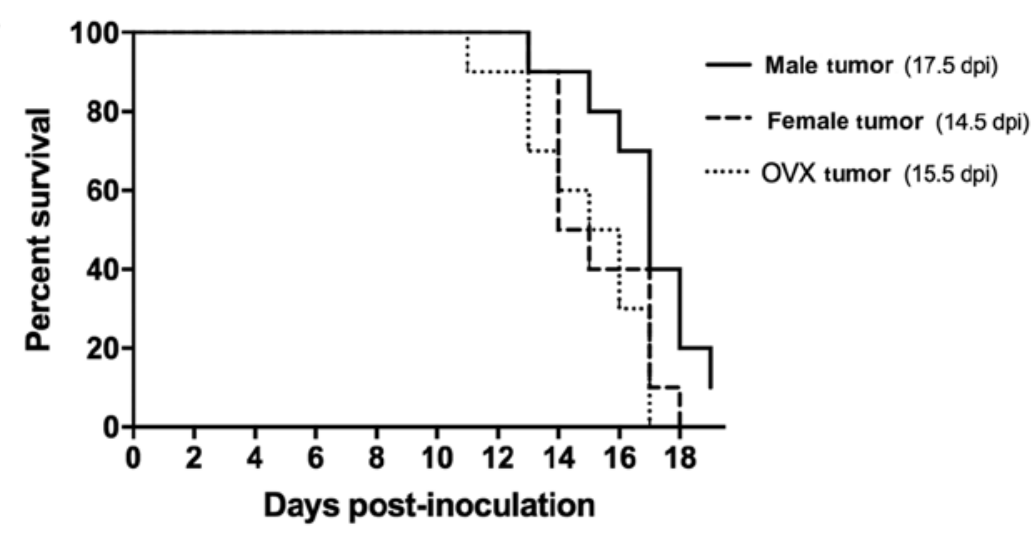

B

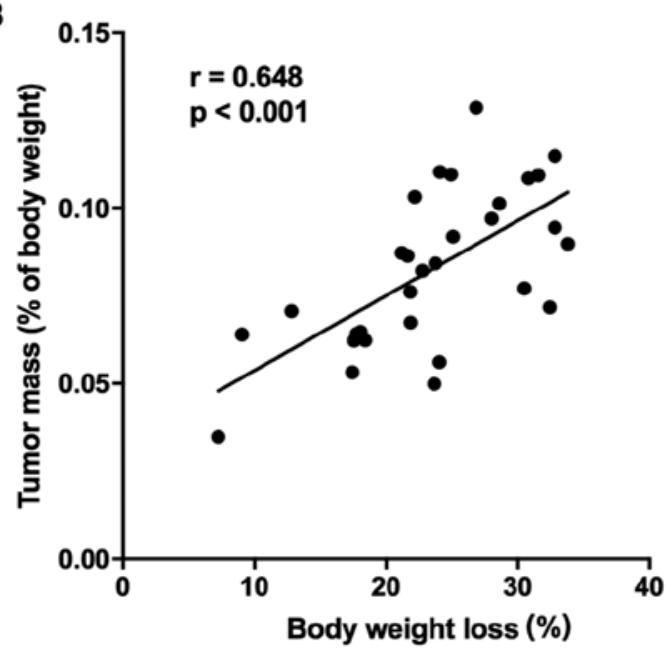

C

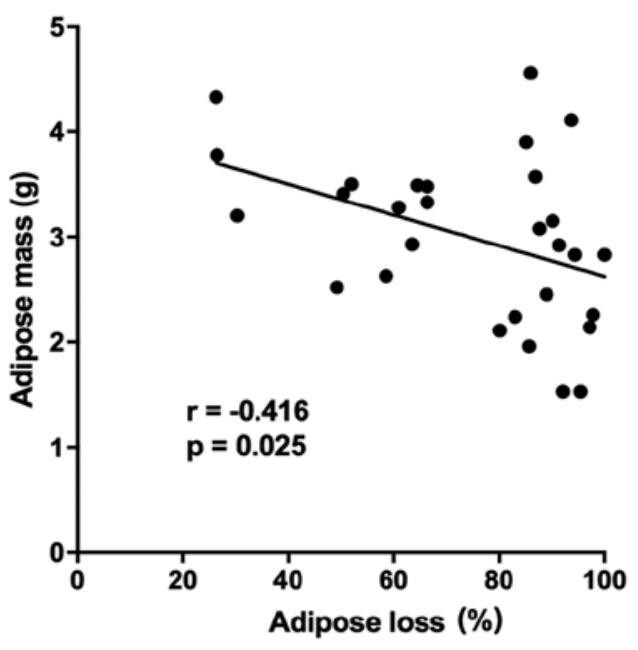

D

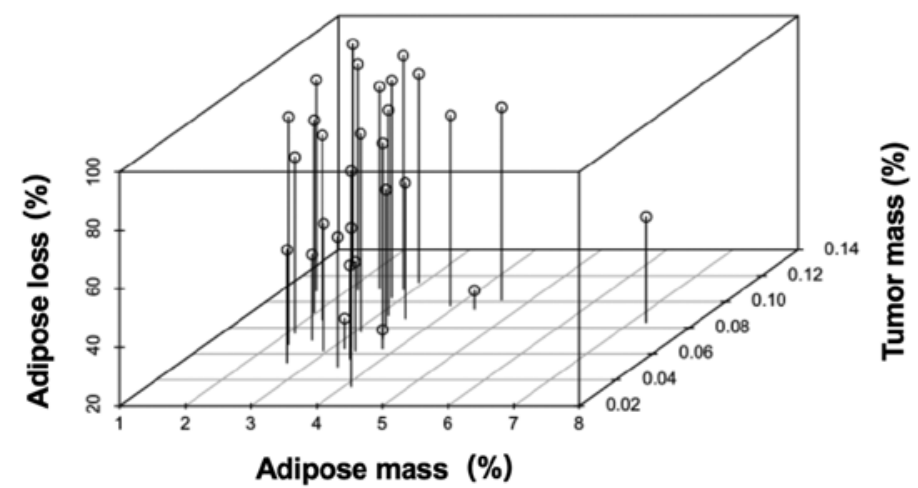

Figure 4. Tumor and adipose mass are predictors of severity of C-26-induced cachexia. (A) Kaplan-Meier survival plot determining incidence of cachexia (defined as $\geq 5 \%$ body weight loss) of tumor mice by study day; the P-value represents a comparison of the 3 groups using a Mantel-Cox test. (B) Relationship between tumor mass (normalized to tumor-free body weight at necropsy) and percentage of peak body weight loss in tumor-bearing mice. (C) Relationship between day 8 EchoMRI adipose mass and percentage of adipose loss in tumor mice. (D) 3-D scatterplot of the relationship between the percentage of tumor mass and day 8 EchoMRI adipose mass ( $\mathrm{g}$ ) on the percentage of adipose loss in tumor mice. $\mathrm{N}=30$ mice total. Correlations between continuous variables were assessed using Pearson's correlation coefficients where $\mathrm{P}<0.05$ was considered statistically significant. C-26, colon-26; Dpi, days post-inoculation.

EchoMRI adipose mass $(\mathrm{g})$ on the percentage of adipose loss, when controlling for the percentage of tumor mass (Table II, $\mathrm{P}=0.09$ ). The estimated effect of a $1-\mathrm{g}$ increase in EchoMRI adipose mass was a 5.9 percentage point decrease in adipose loss. When this multiple linear regression model was stratified by sex/ovariectomy, we found a significant association between tumor mass and adipose loss in female tumor mice (Table III,
$\mathrm{P}=0.003)$ and weaker evidence of association in OVX tumor mice (Table III, $\mathrm{P}=0.113$ ).

\section{Discussion}

Tumor and adipose mass as predictors for severity of cancer cachexia. With the understanding that adiposity may predict 
Table II. Multiple linear regression analysis with adipose mass and percentage of tumor/BW as predictors of percentage of adipose loss in C-26 tumor-bearing mice.

\begin{tabular}{lccc}
\hline Variable & $\begin{array}{c}\text { Estimate } \\
(\beta)\end{array}$ & $\begin{array}{c}\text { Standard } \\
\text { error }\end{array}$ & P-value \\
\hline Intercept & 59.5 & 19.4 & $0.005^{\mathrm{a}}$ \\
Adipose mass $(\mathrm{g})$ & -5.9 & 3.4 & 0.090 \\
Percentage of tumor/BW & 4.0 & 1.6 & $0.022^{\mathrm{a}}$ \\
\hline
\end{tabular}

Indicates statistical significance $\mathrm{P}<0.05$. BW, body weight; C-26, colon-26.

Table III. Multiple linear regression analysis with adipose mass and percentage of the tumor/BW as predictors of percentage of adipose loss in C-26 tumor-bearing mice stratified by sex/ovariectomy.

A, Male

\begin{tabular}{lrrr}
\hline Variable & $\begin{array}{c}\text { Estimate } \\
(\beta)\end{array}$ & $\begin{array}{c}\text { Standard } \\
\text { error }\end{array}$ & P-value \\
\hline Intercept & 108.8 & 33.3 & $0.014^{\mathrm{a}}$ \\
Percentage of tumor/BW & -155.2 & 353.3 & 0.674 \\
Adipose mass $(\mathrm{g})$ & -6.3 & 5.0 & 0.250 \\
\hline
\end{tabular}

B, Female

\begin{tabular}{lrrr}
\hline Variable & $\begin{array}{c}\text { Estimate } \\
(\beta)\end{array}$ & $\begin{array}{c}\text { Standard } \\
\text { error }\end{array}$ & P-value \\
\hline Intercept & -3.4 & 24.3 & 0.892 \\
Percentage of tumor/BW & 821.5 & 176.1 & $0.003^{\mathrm{a}}$ \\
Adipose mass $(\mathrm{g})$ & 1.9 & 4.1 & 0.666 \\
\hline
\end{tabular}

\section{C, OVX}

\begin{tabular}{lrrr}
\hline Variable & $\begin{array}{c}\text { Estimate } \\
(\beta)\end{array}$ & $\begin{array}{c}\text { Standard } \\
\text { error }\end{array}$ & P-value \\
\hline Intercept & 29.2 & 65.8 & 0.671 \\
Percentage of tumor/BW & 1029.3 & 567.8 & 0.113 \\
Adipose mass $(\mathrm{g})$ & -10.9 & 14.1 & 0.464 \\
\hline
\end{tabular}

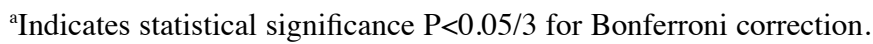
BW, body weight; C-26, colon-26; OVX, ovariectomized.

for survival in cachexia (5), the primary aim of our study was to evaluate the role of adipose tissue changes on outcomes of mice with C-26 adenocarcinoma-induced cachexia. A Kaplan-Meier survival curve analysis was performed to determine the time to incidence of cachexia ( $\geq 5 \%$ body weight loss). We found that both female and OVX tumor mice developed cachexia sooner than male tumor mice (Fig. 4A). One likely explanation for this finding may be related to tumor mass, which was significantly correlated to the percentage of body weight loss in mice, regardless of sex or ovariectomy (Fig. 4B). Another possible explanation for the earlier incidence of cachexia in female and OVX tumor mice could be explained by low adipose mass as a predictor of C-26-induced weight loss. We found that tumor mice having less adipose mass (calculated through EchoMRI at day 8 post-inoculation, before onset of cachexia) was associated with higher percentage of adipose loss (Fig. 4C), but not the percentage of body weight loss (data not shown), at the end of the study. The discrepancy of findings between the percentage of adipose loss and the percentage of body weight loss could be explained by relatively minute changes in lean body mass masking the pronounced adipose wasting observed (Fig. 1E and F). Corroborating the idea that having more adipose mass may be protective in cancer cachexia, OVX mice in the $A p c^{\mathrm{Min} /+}$ model were also protected from body weight loss during cachexia due to their transient weight gain after ovariectomy (29). The earlier onset of cachexia in female and OVX tumor mice in our study may have had an impact on their muscle function, as suggested by the trend of decreased forelimb grip strength at the end of the study in comparison to male tumor mice (Fig. 2E). It should be noted that for 2 female and 2 OVX tumor mice euthanized early due to signs of moribundity, final grip strength measurements were not obtained.

To distinguish between the effects of tumor mass and adipose mass on cachexia independently, we performed multiple linear regression correlating these variables to the percentage of adipose loss in mice (Table II and Fig. 4D). We found a significant effect of tumor mass on the percentage of adipose loss $(\mathrm{P}=0.022)$ when adjusting for EchoMRI day 8 adipose mass $(\mathrm{g})$. However, there was weaker evidence $(\mathrm{P}=0.09)$ for the effect of EchoMRI day 8 adipose mass $(\mathrm{g})$ on the percentage of adipose loss, when accounting for tumor mass. A stratified analysis by sex/ovariectomy suggests that it is the association in the female tumor group (and to a lesser extent the OVX tumor group) that is driving the association between tumor mass and adipose loss (Table III). However, ANOVA did not detect a statistically significant interaction between sex/ovariectomy and tumor mass in predicting adipose loss $(\mathrm{P}=0.064)$. To the best of our knowledge, the impact of initial adipose mass predicting for weight loss at the onset of cachexia studies in mice has not been addressed. This is a fundamental question of the 'obesity paradox', which postulates that excess adiposity may counterintuitively confer survival in specific populations of individuals with chronic diseases and cancer $(8,9)$. To directly address whether having excess adipose could improve outcomes of cancer cachexia, a future study should evaluate mouse models of obesity in the C-26 adenocarcinoma and other models of cancer cachexia.

Effect of sex status in cancer cachexia. The secondary aim of our study was to evaluate the role of sex status on outcomes of mice with C-26 adenocarcinoma-induced cachexia. Many, but not all clinical studies report a higher incidence of cancer cachexia in men than women (10-13). Additionally, male cancer patients lose weight at a faster rate than female patients, which contributes to impaired quality of life and shorter survival time after initial diagnosis (12,30-32). These findings indicate 
that sex may play a role in cancer cachexia development and progression. However, pre-clinical studies elucidating sex differences in cancer cachexia are scarce $(29,33,34)$. A study using the C-26 model found that estrogen receptor signaling in female mice was responsible for reducing the severity of cancer cachexia in comparison to male mice (33). In the genetic $A p c^{\mathrm{Min} /+}$ model of cancer cachexia, lower circulating IL-6 was partially responsible for the reduced severity of cancer cachexia in female mice, although the study did not evaluate if estrogen was differentially regulating IL-6 function (34). Notably, the initiation of acyclicity (e.g., loss of estrogen cycling) brought on by cachexia, but not ovariectomy was a predictor of cachexia severity in this mouse model (29).

In the present study, female and OVX tumor mice experienced body weight loss (Fig. 1B) and muscle loss (Fig. 2A) similar to male tumor mice, which is in contrast to other studies showing less muscle wasting in female mice $(29,33,34)$. These previous studies did not observe a difference in tumor burden between male and female mice, whereas we found that female tumor mice had larger tumors when expressed as a percentage of their necropsy body weight (Fig. 1C). One possible explanation for this could be the tumor growth-promoting effects of estrogen (35), although to our knowledge, the sensitivity of C-26 cells to estrogen has not been tested. Female and OVX tumor mice exhibited adipose loss similar to male tumor mice; however, female tumor mice lost significantly more adipose than OVX tumor mice (Fig. 1F). This observation could be predicted by at least 2 factors associated with cachexia, e.g., higher tumor mass and lower adipose mass. Female tumor mice had significantly smaller heart and spleen masses than male tumor mice (Fig. 3B) at the end of the study; this difference could possibly be attributed to initial body weight differences and lower lean masses (measured through EchoMRI) in female tumor mice at day 0 compared to male tumor mice (data not shown). Quadriceps mRNA markers of proteolysis and apoptosis were also similar between the 3 tumor groups. While Hetzler et al observed lower plasma IL-6 in female mice of the $A p c^{M i n /+}$ model of cancer cachexia (34), we did not find differences in plasma IL-6 between sexes of C-26 tumor-bearing mice (Fig. 3C). Moreover, we did not observe an association between plasma IL-6 with body weight loss or tumor mass in any of the tumor groups (data not shown). Plasma adiponectin was significantly decreased in all 3 tumor groups in comparison to their respective controls (Table I); however, OVX tumor mice had significantly higher, while male tumor mice and female tumor mice had significantly lower adiponectin. However, we did not find any relationship between plasma adiponectin with plasma IL-6 or body weight loss in the 3 tumor groups (data not shown). Overall, our results indicated that female and OVX tumor mice respond to $\mathrm{C}-26$-induced cachexia similarly to their male counterparts.

Strengths and limitations. A major strength of this study is the extensive characterization of male, female and OVX mouse response to $\mathrm{C}-26$ cachexia by measuring key physiological outcomes beyond body weight loss and tumor mass. These include measurements of body composition and food intake over time, plasma markers, muscle fiber cross-sectional area, and muscle grip strength. In addition, this study utilized Kaplan-Meier survival curve analysis to elucidate differences in progression of cachexia, as well as multiple linear regression modeling to find key predictors (i.e., tumor mass and adipose mass) of body wasting independent of sex and ovariectomy. A limitation of the current study is that initial body weights of female and OVX mice were not equal to that of males at the beginning of the study, thus introducing body weight and adiposity as a variable that may have influenced outcomes such as tumor mass. Additionally, estrogen and testosterone levels were not measured in this study, thus we could not extensively explore their influences on skeletal muscle metabolism. As with other rodent models of cancer cachexia, the C-26 model has its limitations. Foremost is that the C-26 model is a xenograft model whereby tumor cells are transplanted into mice, while human cancers arise from spontaneous genetic mutations (36). Another key difference is the timing and progression of cachexia, since C-26 mice often develop cachexia rapidly within 3 weeks of inoculation to achieve a final tumor mass up to $10 \%$ of body weight. In contrast, humans generally develop cachexia over the span of months, and have much smaller ratio of tumor to body weight burden. Despite these limitations, the C-26 model currently remains a useful and efficient model to study the pathogenesis of cancer cachexia.

Overall, this study revealed that higher tumor mass and lower adipose mass are key predictors of cancer cachexia in the $\mathrm{C}-26$ adenocarcinoma model. The role of sex status may be subtle as female and OVX mice develop symptoms of C-26 adenocarcinoma-induced cancer cachexia similarly to male mice. To directly address the potential protective role of adipose mass in cancer cachexia, a future study should assess outcomes of cancer cachexia in obese vs. lean mice.

\section{Acknowledgements}

The authors acknowledge Brad Cotten for assistance throughout the animal study and thank Erin Talbert for technical support and scholarly discussions about the data.

\section{Funding}

This research was supported by NIHR21CA185140 and support of TB on pre-doctoral fellowship NINDS T32NS077984-05.

\section{Availability of data and materials}

The data sets used during the current study are available from the corresponding author on reasonable request.

\section{Authors' contributions}

TB and MAB contributed to the conception and design of the study. TB, DS, RMC, and AA contributed to the collection of data and generation of results. TB and PMS contributed to the statistical analyses of the results. All authors contributed to the interpretation of data and results. TB contributed to the majority of manuscript writing and all authors critically revised it for important intellectual content. All authors approved the final manuscript to be published and agree to be accountable for all aspects of the manuscript. 


\section{Ethics approval and consent to participate}

Experiments involving mice were approved by The Ohio State University Institutional Animal Care and Use Committee.

\section{Patient consent for publication}

Not applicable.

\section{Competing interests}

The authors declare that they have no competing interests.

\section{References}

1. Fearon K,StrasserF,AnkerSD,Bosaeus I,BrueraE,Fainsinger RL, Jatoi A, Loprinzi C, MacDonald N, Mantovani G, et al: Definition and classification of cancer cachexia: An international consensus. Lancet Oncol 12: 489-495, 2011.

2. Tisdale MJ: Cachexia in cancer patients. Nat Rev Cancer 2: 862-871, 2002

3. Fouladiun M, Korner U, Bosaeus I, Danaeryd P, Hyltander A and Lundholm KG: Body composition and time course changes in regional distribution of fat and lean tissue in unselected cancer patients on palliative care - correlations with food intake, metabolism, exercise capacity, and hormones. Cancer 103: 2189-2198, 2005.

4. Dahlman I, Mejhert N, Linder K, Agustsson T, Mutch DM, Kulyte A, Isaksson B, Permert J, Petrovic N, Nedergaard J, et al: Adipose tissue pathways involved in weight loss of cancer cachexia. Br J Cancer 102: 1541-1548, 2010.

5. Di Sebastiano KM, Yang L, Zbuk K, Wong RK, Chow T, Koff D, Moran GR and Mourtzakis M: Accelerated muscle and adipose tissue loss may predict survival in pancreatic cancer patients: The relationship with diabetes and anaemia. Br J Nutr 109 302-312, 2013.

6. Ebadi M and Mazurak VC: Evidence and mechanisms of fat depletion in cancer. Nutrients 6: 5280-5297, 2014.

7. Agustsson T, Ryden M, Hoffstedt J, van Harmelen V, Dicker A, Laurencikiene J, Isaksson B, Permert J and Arner P: Mechanism of increased lipolysis in cancer cachexia. Cancer Res 67: 5531-5537, 2007.

8. Kalantar-Zadeh K, Horwich TB, Oreopoulous A, Kovesdy CP, Younessi H, Anker SD and Morley JE: Risk factor paradox in wasting diseases. Curr Opin Clin Nutr Metab Care 10: 433-442, 2007.

9. Martin L, Birdsell L, Macdonald N, Reiman T, Clandinin MT, McCargar LJ, Murphy R, Ghosh S, Sawyer MB and Baracos VE: Cancer cachexia in the age of obesity: Skeletal muscle depletion is a powerful prognostic factor, independent of body mass index. J Clin Oncol 31: 1539-1547, 2013.

10. Nakamura N, Hara T, Shibata Y, Matsumoto T, Nakamura H, Ninomiya S, Kito Y, Kitagawa J, Kanemura N and Goto N: Sarcopenia is an independent prognostic factor in male patients with diffuse large B-cell lymphoma. Ann Hematol 94: 2043-2053, 2015.

11. Kim EY, Kim YS, Park I, Ahn HK, Cho EK and Jeong YM: Prognostic significance of CT-determined sarcopenia in patients with small-cell lung cancer. J Thorac Oncol 10: 1795-1799, 2015.

12. Wallengren O, Iresjo BM, Lundholm K and Bosaeus I: Loss of muscle mass in the end of life in patients with advanced cancer. Support Care Cancer 23: 79-86, 2015.

13. Masel EK, Berghoff AS, Fureder LM, Heicappell P, Schlieter F, Widhalm G, Gatterbauer B, Dieckmann U, Birner P, Bartsch R, et al: Decreased body mass index is associated with impaired survival in lung cancer patients with brain metastases: A retrospective analysis of 624 patients. Eur J Cancer Care (Engl) 26: 6, 2017.

14. Karastergiou K, Smith SR, Greenberg AS and Fried SK: Sex differences in human adipose tissues - the biology of pear shape. Biol Sex Differ 3: 13, 2012.
15. Carr MC: The emergence of the metabolic syndrome with menopause. J Clin Endocrinol Metab 88: 2404-2411, 2003.

16. Aulino P, Perardi E, Cardillo VM, Rizzuto E, Perniconi B, Ramina C, Padula F, Spugnini EP, Baldi A, Faiola F, et al: Molecular, cellular and physiological characterization of the cancer cachexia-inducing C26 colon carcinoma in mouse. BMC Cancer 10: 363, 2010.

17. Strassmann G, Fong M, Kenney JS and Jacob CO: Evidence for the involvement of interleukin 6 in experimental cancer cachexia. J Clin Invest 89: 1681-1684, 1992.

18. Tisdale MJ: Mechanisms of cancer cachexia. Physiol Rev 89: 381-410, 2009.

19. Murphy KT, Chee A, Trieu J, Naim T and Lynch GS: Importance of functional and metabolic impairments in the characterization of the C-26 murine model of cancer cachexia. Dis Model Mech 5: 533-545, 2012.

20. Tian M, Asp ML, Nishijima Y and Belury MA: Evidence for cardiac atrophic remodeling in cancer-induced cachexia in mice. Int J Oncol 39: 1321-1326, 2011.

21. Johns N, Stephens NA and Fearon KC: Muscle wasting in cancer. Int J Biochem Cell Biol 45: 2215-2229, 2013.

22. Kliewer KL, Ke JY, Tian M, Cole RM, Andridge RR and Belury MA: Adipose tissue lipolysis and energy metabolism in early cancer cachexia in mice. Cancer Biol Ther 16: 886-897, 2015.

23. Livak KJ and Schmittgen TD: Analysis of relative gene expression data using real-time quantitative PCR and the 2(-Delta Delta C(T)) method. Methods 25: 402-408, 2001.

24. Silva KA, Dong J, Dong Y, Dong Y, Schor N, Tweardy DJ, Zhang L and Mitch WE: Inhibition of Stat 3 activation suppresses caspase- 3 and the ubiquitin-proteasome system, leading to preservation of muscle mass in cancer cachexia. J Biol Chem 290: 11177-11187, 2015.

25. Zhou X, Wang JL, Lu J, Song Y, Kwak KS, Jiao Q, Rosenfeld R, Chen Q, Boone T, Simonet WS, et al: Reversal of cancer cachexia and muscle wasting by ActRIIB antagonism leads to prolonged survival. Cell 142: 531-543, 2010.

26. Asp ML, Tian M, Kliewer KL and Belury MA: Rosiglitazone delayed weight loss and anorexia while attenuating adipose depletion in mice with cancer cachexia. Cancer Biol Ther 12: 957-965, 2011.

27. Talbert EE, Metzger GA, He WA and Guttridge DC: Modeling human cancer cachexia in colon 26 tumor-bearing adult mice. J Cachexia Sarcopenia Muscle 5: 321-328, 2014.

28. Brochu-Gaudreau K, Rehfeldt C, Blouin R, Bordignon V, Murphy BD and Palin MF: Adiponectin action from head to toe. Endocrine 37: 11-32, 2010.

29. Hetzler KL, Hardee JP, LaVoie HA, Murphy EA and Carson JA: Ovarian function's role during cancer cachexia progression in the female mouse. Am J Physiol Endocrinol Metab 312: E447-E459, 2017.

30. Palomares MR, Sayre JW, Shekar KC, Lillington LM and Chelebowski RT: Gender influence on weight-loss pattern and survival of nonsmall cell lung carcinoma patients. Cancer 78: 2119-2126, 1996.

31. Kimura M, Naito T, Kenmotsu H, Taira T, Wakuda K, Oyakawa T, Hisamatsu Y, Tokito T, Imai H, Akamatsu H, et al: Prognostic impact of cancer cachexia in patients with advanced non-small cell lung cancer. Support Care Cancer 23: 1699-1708, 2015.

32. Stephens NA, Gray C, MacDonald AJ, Tan BH, Gallagher IJ, Skipworth RJ, Ross JA, Fearon KC and Greig CA: Sexual dimorphism modulates the impact of cancer cachexia on lower limb muscle mass and function. Clin Nutr 31: 499-505, 2012.

33. Cosper PF and Leinwand LA: Cancer causes cardiac atrophy and autophagy in a sexually dimorphic manner. Cancer Res 71: 1710-1720, 2011.

34. Hetzler KL, Hardee JP, Puppa MJ, Narsale AA, Sato S, Davis JM and Carson JA: Sex differences in the relationship of IL-6 signaling to cancer cachexia progression. Biochim Biophys Acta 1852: 816-825, 2015.

35. McGuire WL, Horwitz KB, Chamness GC and Zava DT: A physiological role for estrogen and progesterone in breast cancer. J Steroid Biochem 7: 875-882, 1976.

36. Richmond A and Su Y: Mouse xenograft models vs GEM models for human cancer therapeutics. Dis Model Mech 1: 78-82, 2008. 